\title{
EDITORIAL
}

\section{Targeting Glia for Treatment of Neurological Disease}

Translational research in neurological disease has focused on neuronal disorders. Yet, the function of glia is indispensable for proper neuronal survival and CNS function. Some of the most prominent supportive functions of astrocytes include glutamate uptake, water transport, control of cerebral blood flow, buffering of ions, and neurotrophic support (see Kimelberg and Nedergaard, page 338). In recent years, astrocytes have also been shown to play an integral role in complex cognitive functions, including sensory processing and sleep (see Kimelberg and Nedergaard, page 338). Similarly, microglia play critical roles for regulating immune responses in the brain and provide neurotrophic support. Given these pleiotropic functions of astrocytes and microglia, it is not surprising that their dysfunction is an important contributor to multiple neurological diseases. In fact, in some cases, astrocyte dysfunction is the primary basis of the disease (see Messing, LaPash Daniels, and Hagemann, page 507). Although initially neglected, the examination of glia is now flourishing and providing valuable new insight into the function and dysfunction of glia during disease. As such, opportunities are emerging by which astrocyte and microglial function might be targeted to suppress progression of the disease. Following a detailed discussion of normal physiological functions of astrocytes (see Kimelberg and Nedergaard, page 338), this issue of Neurotherapeutics will explore the role of astrocytes and microglia in specific disease states and highlight potential therapeutic opportunities. It is our hope that these articles will help to spark interest, research, and funding directed at targeting glia for future treatment of neurological disease.

The neurotoxic factors released by activated microglia are important contributors to neuronal death and dysfunction in neurological disease. In this issue, the contributions of microglia to neurodegenerative disease (see Lull and Block, page 354), traumatic brain injury (see Loane and Byrnes, page 366), stroke (see Yenari, Kauppinen, and Swanson, page 378), and HIV-associated neurocognitive disease (HAND) (see Gelbard et al., page 392) are explored. In the case of neurodegenerative diseases, the pathological processes that contribute to neuronal death and dysfunction extend over multiple years. Mirroring this time course, chronic activation of microglia is present in neurodegenerative diseases (see Lull and Block, page 354). Interestingly, this chronic activation may be induced by either multiple stimuli or a single stimulus. Thus, the effects of an injurious stimulus may propagate through the actions of microglia, contributing to neuronal death over extended time periods. Of course, microglia are not simply harbingers of neuropathological processes. In contrast, during acute injury, their function as the resident immunological cell in the brain may be an important contribution to neuronal survival. This paradox of the "good and bad" functions of microglia in traumatic brain injury and spinal cord injury presents an important paradox to consider when targeting microglial function (see Loane and Byrnes, page 366). Similarly, the role of microglia in stroke, the third leading cause of death in the U.S., is complex. In the acute phase of stroke, microglia contribute importantly to cell death. The various stimuli that activate microglia, the signal transduction pathways that contribute to microglial activation, and the mechanisms of microglial cytotoxicity in stroke are discussed in detail in the review by Yenari, Kauppinen, and Swanson (page 378). Interestingly, therapeutic agents that reduce microglial activation during stroke, such as minocycline, are being entered into clinical trials for stroke. However, as the authors stress, it will be important to develop specific therapies that diminish the pathological functions of microglia while maintaining their salutary effects. HAND contributes significantly to the morbidity of HIV, and its impact remains significant even in patients treated with retroviral therapy (see Gelbard et al., page 392). Interestingly, in patients with HIV, the mixed lineage kinase type 3 (MLK3) is pathologically activated in microglia, as well as other cell types in the brain. This activation of MLK3 leads to neuronal dysfunction. Translational research is identifying small molecule inhibitors of MLK3 as a potential treatment for HAND.

Astrocytes significantly contribute to neuronal health in the neurodegenerative diseases, including Alzheimer's disease (AD) (see Verkhratsky et al., page 399), Parkinson's disease (PD) (see Rappold and Tieu, page 413), and amyotrophic lateral sclerosis (ALS) (see Vargas and Johnson, page 471). In $\mathrm{AD}$, astrocytes influence $\beta$-amyloid abundance in the extracellular space, are involved in the neuroinflammatory response to $\beta$-amyloid, and likely impact the neurovascular dysfunction observed in AD. In the case of PD, astrocytes play dual roles, which may be helpful or harmful (see Rappold and Tieu, page 413). Astrocytes likely improve dopaminergic neuronal survival by uptake of toxic molecules, release of trophic 
factors, and providing antioxidant defenses. Yet, astrocytes also release pro-inflammatory molecules that may damage dopamine neurons. Uptake of glutamate is a primary and critical function of astrocytes. Because excitotoxicity is a key mediator of motor neuron death in ALS, it is not surprising that astrocytes may be a significant determinant of neuronal death in this disease (see Vargas and Johnson, page 471). Moreover, recent work has demonstrated that astrocytes expressing mutant superoxide dismutase, which is responsible for familial ALS, cannot support the health of motor neurons. ${ }^{1}$ This observation suggests a central role for astrocytes in the development on motor neuron cell death in ALS.

One of the recurrent themes presented in the discussions concerning astrocyte function in disease and difficulties in targeting astrocytes for treatment of neurological disease is the fact that astrocytes may serve both adaptive and pathological functions. Astrocyte gliosis is observed in most neurological diseases and is a good example of this dilemma (see Hamby and Sofroniew, page 494). For example, beneficial aspects of astrocyte gliosis are their uptake of excitotoxic glutamate, antioxidant effects, release of neuroprotective agents, and their ability to limit the spread of infectious agents. Yet, reactive astrocytes may release inflammatory cytokines, produce reactive oxygen species, and the glial scar could impede axonal growth. These divergent properties may manifest, depending on the severity of injury and the presence/absence of other signaling molecules in the extracellular milieu. Adding another complication to these divergent actions of astrocytes is that, in acute neurological disease, such as stroke, the function of particular molecules secreted by astrocytes (or microglia-see Yenari, Kauppinen, and Swanson, page 378) may be detrimental during some phases of stroke yet advantageous during others (see Zhao and Rempe, page 439). For example, vascular endothelial growth factor (VEGF) and matrix metalloproteinase inhibitors (MMPs) increase edema and stroke volume when present acutely after stroke onset. In contrast, during the days and weeks after stroke, VEGF and MMP expression by astrocytes (and other cell types) is critical for angiogenesis and recovery from stroke. ${ }^{2}$ Therefore, the potential targeting of astrocyte and microglial function in acute neurological disease will need to consider these temporal specific functions of glia and the molecules they secrete.

One approach to target astrocyte function for neurological disease treatment is to design treatment modalities that enhance or emulate the "good" functions of astrocytes. For example, multiple studies have administered astrocyte-derived neuroprotective factors, such as glial-derived neurotrophic factor (GDNF) and erythropoietin (EPO), in the hopes of enhancing neuroprotection in neurological disease. GDNF effectively reduces neuronal death in animal models of PD, ALS, and stroke (see Rappold and Tieu, page 413; Vargas and Johnson, page 471; and Zhao and Rempe, page 439). Although GDNF was attempted in human trials of PD, side effects and conflicting results diminished enthusiasm for GDNF as a potential treatment (see Rappold and Tieu, page 413). Similarly, EPO is a neuroprotectant released by astrocytes, and it reduces stroke volume in animal models of stroke. While phase 2 clinical trials of EPO in stroke patients appeared promising, a recent phase 3 trial failed to show efficacy. ${ }^{3}$ Given the relative lack of permeability of the blood-brain barrier for these neurotrophic proteins, a more promising approach may be to develop small molecules that induce expression of these neuroprotectants in endogenous astrocytes, as opposed to the exogenous delivery of the neurotrophin. Another example of targeting the "good" function of astrocytes includes enhancing astrocyte glutamate uptake. In fact, $\beta$-lactam antibiotics (Ceftriaxone) effectively increase expression of glutamate transporters in cultured astrocytes and delay motor neuron loss in animal models of ALS (see Vargas and Johnson, page 471). Given that these antibiotics have a long history of clinical use, it will be interesting to determine whether these agents are effective when tested in clinical trials. A second approach to target astrocyte function for neurological disease treatment is to reduce the "bad" functions of astrocytes. Arundic acid (AA; ONO-2506) is a compound identified on a chemical screen in astrocyte cultures that reduces the production of the neurotoxicant $S-100 \beta$ by activated astrocytes. ${ }^{4}$ This compound is currently being studied in clinical trials for treatment of ALS (see Vargas and Johnson, page 471), but was not effective for treatment of stroke (see Zhao and Rempe, page 439).

Beyond the treatment of neurodegenerative disease and stroke, astrocyte function also importantly contributes to epilepsy (see de Lanerolle, Lee, and Spencer, page 424), chronic pain (see Gao and Ji, page 482), and hepatic encephalopathy (see Brusilow et al., page 452). In epilepsy, the reduction of glutamine synthase in astrocytes may be of particular relevance to glutamate homeostasis. Moreover, the epileptic hippocampus is likely enriched with NG2-like glia, which could make important contributions to the spread of excitability in the hippocampus. In chronic pain, the release of pro-inflammatory cytokines by astrocytes is an important contributor to this disease state. Encouragingly, targeting these astrocyte-supported inflammatory signaling pathways diminishes chronic pain in animal models. In the syndrome of hepatic encephalopathy, the accumulation of ammonia is central to the pathological state (see Brusilow et al., page 452). Hyperammonemia leads to the accumulation of glutamine in astrocytes and astrocyte swelling, which are likely important contributors to astrocyte dysfunction and the hepatic encephalopathy. Targeting glutamine synthesis from ammonia is proposed as a potential mech- 
anism for treating this disorder (see Brusilow et al., page 452).

Finally, one disease of which astrocyte dysfunction is the principal cause is Alexander's disease, a rare and fatal disorder of the CNS (see Messing, LaPash Daniels, and Hagemann, page 507). Alexander's disease is caused by mutations in the genetic sequence of the gene expressing glial fibrillary acidic protein (GFAP). This mutant GFAP acts in an autosomal dominant fashion. As such, current translational research is exploring compounds that may reduce GFAP expression. In addition, the antioxidant effects of astrocytes may be enhanced or glutamate uptake increased as potential approaches for treatment of Alexander's disease.

While this issue of Neurotherapeutics explores translational research that targets astrocyte and microglial function, glia interact in concert with neurons and the vasculature to sculpt the response to disease. As such, no therapeutic intervention or pharmacological manipulation of astrocytes/microglia can be assumed to solely affect astrocytes/microglia instead of neurons or vice versa. Although the intracellular signaling pathways in neurons and astrocytes are very different, they express receptors for many of the same transmitters. The effect of receptor activation, however, differs fundamentally in neurons and astrocytes. For example, glutamate triggers depolarization, generation of action potentials, and excitotoxic injury of neurons. In contrast, astrocytes display oscillatory and propagated $\mathrm{Ca}^{2+}$ signals in response to glutamate, and their membrane potential and viability are not affected by prolonged exposure to high glutamate concentration. Thus, targeting disease processes in these glia cells may have opposing, compensatory, or off-target effects in neurons or the vasculature, diminishing the intended therapeutic benefit. As such, future work will need to consider the complex interactions of these multiple cell types as we search for more efficacious treatments for these burdensome diseases.

David A. Rempe, M.D., Ph.D. Department of Neurology, Stroke Division Center for Neural Development and Disease University of Rochester School of Medicine and Dentistry Rochester, New York Maiken Nedergaard, M.D., Ph.D. Division of Glia Disease and Therapeutic Center for Translational Neuromedicine Department of Neurosurgery

University of Rochester School of Medicine and Dentistry Rochester, New York

\section{REFERENCES}

1. Nagai M, Re DB, Nagata T, et al. Astrocytes expressing ALS-linked mutated SOD1 release factors selectively toxic to motor neurons. Nat Neurosci 2007;10:615-622.

2. Zhao BQ, Wang S, Kim HY, et al. Role of matrix metalloproteinases in delayed cortical responses after stroke. Nat Med 2006;12: 441-445.

3. Ehrenreich H, Weissenborn K, Prange H, et al. Recombinant human erythropoietin in the treatment of acute ischemic stroke. Stroke 2009;40:e647-e656.

4. Asano T, Mori T, Shimoda T, et al. Arundic acid (ONO-2506) ameliorates delayed ischemic brain damage by preventing astrocytic overproduction of S100B. Curr Drug Targets CNS Neurol Disord 2005;4:127-142. 